\title{
Phenotypic and behavioral variability within Angelman Syndrome group with UPD
}

\author{
Cintia Fridman ${ }^{1}$, Monica C. Varela ${ }^{1}$, Kette Valente $^{2}$, Maria J. Marques-Dias ${ }^{3}$ and Célia P. Koiffmann ${ }^{1}$ \\ ${ }^{1}$ Centro de Estudos do Genoma Humano, Departamento de Biologia, Instituto de Biociências, \\ Universidade de São Paulo, São Paulo, Brazil. \\ ${ }^{2}$ Laboratório de Neurofisiologia Clínica do Instituto de Psiquiatria, Hospital das Clínicas, \\ FMUSP, São Paulo, Brazil. \\ ${ }^{3}$ Depto de Pediatria, Instituto da Criança, Hospital das Clínicas, FMUSP, São Paulo, Brazil.
}

\begin{abstract}
The Angelman syndrome (AS) (developmental delay, mental retardation, speech impairment, ataxia, outbursts of laughter, seizures) can result either from a 15q11-q13 deletion, or from paternal uniparental disomy (UPD), imprinting, or UBE3A mutations. We describe here the phenotypic and behavioral variability detected in eight UPD patients out of a group of 58 AS patients studied. All of them presented developmental delay, mental retardation, ataxia, speech impairment, and frequent drooling. Only one had microcephaly, whereas in two of them the OFC (head circumference) was above the $98^{\text {th }}$ percentile. The weight of all patients was above the $50^{\text {th }}$ percentile, and in three of them the height was above the $90^{\text {th }}$ percentile. Three were able to say a few words and to communicate by gestures. Two patients presented hyperphagia, and three presented skin picking, common features in the Prader-Willi syndrome (PWS). Four patients (4/7) had wide-spaced teeth. Five presented seizures, and two others did not manifest frequent laughter. One patient was very different from the others, as he showed a better understanding and abilities to communicate, to play video games and to draw. We suggest here that there seems to be an extreme phenotypic and behavioral variability within the UPD group, and that both typical patients and those with mental retardation, language impairment, happy disposition, and hyperactivity should be tested for AS.
\end{abstract}

Key words: Angelman syndrome, uniparental disomy, macrosomy, macrocephaly.

Received: May 6, 2002; accepted: June 18, 2002.

\section{Introduction}

Angelman syndrome (AS) (Angelman, 1965) is characterized by hypotonia, severe mental retardation, absent speech, seizures, ataxia, outbursts of laughter, micro and/or brachycephaly, macrostomia, and prognathism. The gait is described as wide-based with arms held flexed and upheld at the elbows (Clayton-Smith and Pembrey, 1992; Fryburg et al., 1991; Robb et al., 1989).

Approximately $70-75 \%$ of individuals with AS have 15q11-q13 deletions which are of maternal origin (Knoll et al., 1989; Magenis et al., 1990). Furthermore, paternal uniparental disomy (UPD) of chromosome 15 is found in about 2-3\% of the patients (Driscoll, 1994; Magenis et al., 1990). About $1-5 \%$ of patients have biparental inheritance of chromosome 15, but show abnormal methylation pattern and gene expression. These patients have a mutation in the

Send correspondence to C.P. Koiffmann. Depto de Biologia, Instituto de Biociências, USP, Caixa Postal 11.461, 05422-970 São Paulo, SP, Brazil. E-mail: cpkoiffm @ib.usp.br. imprinting center (Buiting et al., 1995; Dittrich et al., 1996; Ohta et al., 1999; Saitoh et al., 1996). In addition, there is a class of patients $(\sim 8 \%)$ with mutations in the UBE3A gene (Kishino et al., 1997; Malzac et al., 1998; Matsuura et al., 1997).

In a previous study (Fridman et al., 2000a), we described the clinical and behavioral manifestations of 4 cases of paternal UPD15 among Brazilian AS children, and compared these cases to UPD cases from the literature (Bottani et al., 1994; Freeman et al., 1993; GillessenKaesbach et al.,1995; Malcolm et al., 1991; Nicholls et al., 1992; Prasad and Wagstaff, 1997; Smeets et al., 1992; Smith et al., 1997, 1998). We also compared the data with those of our deletion patients $(n=21)$. We concluded that better speech development, weight above the 75 th percentile and OFC (head circumference) in the upper normal range are characteristics that should be added to the spectrum of clinical variability present in the Angelman syndrome. 
In this paper, we compare the phenotypic variability showed by our UPD group consisting of the patients mentioned above (Fridman et al., 2000a) plus 4 new cases, and present the unexpected features showed by an AS patient with paternal isodisomy.

\section{Subjects and Methods}

\section{Patients}

The eight UPD patients ( 4 boys and 4 girls, ranging in age from 2 ys 7 mo to 21 years) with Angelman syndrome were detected in a group of $58 \mathrm{AS}$ patients diagnosed in our laboratory and referred by neurologists of the Hospital das Clínicas, School of Medicine, University of São Paulo, Brazil.

\section{DNA analysis}

The patients were diagnosed by methylation analysis of SNURF-SNRPN exon 1 (data not shown).

Microsatellite analyses were performed with 3 markers within the critical region 15q11-q13, 4-3RCA (D15S11), LS6-1CA (D15S113), and GABRB3CA (GABRB3). Five loci outside the PWS/AS region (D15S131, D15S984 CYP19, D17S117 and D15S115) were studied to distinguish between deletion and UPD (data not shown), and to detect crossover regions. Loci D15S541 and D15S542, located close to the centromere, were analyzed to determine the meiotic origin of the nondisjunction (Robinson et al., 1998). Multiplex PCR and polyacrylamide gel electrophoresis of ${ }^{32} \mathrm{P}$ end-labeled amplification products followed the method described by Mutirangura et al. (1993).

\section{Results}

Patients $\# 2, \# 3, \# 6, \# 7$, and \#8 were typical AS children with developmental delay, speech impairment, ataxia, happy disposition, and a wide mouth.

Patient \#1 was referred to us years ago, and at that time he was diagnosed as having an overgrowth syndrome and a 15;15 translocation (Wajntal et al., 1993). Later on, he was tested with the methylation assay, because he showed some AS features, such as: absence of speech, ataxia, outbursts of laughter, late-onset seizures, and also hyperphagia, obesity, behavioral problems and skin picking (features commonly seen in PWS patients). In addition, his weight, length and OFC were above the $90^{\text {th }}$ percentile (Fridman et al., 1998).

Patient \#4 was clinically diagnosed as having PWS at the age of 3, based on his history of neonatal hypotonia, poor sucking, developmental delay, obesity and absence of speech. His weight and height were above the $97^{\text {th }}$ percentile.
Patient \#5, although having developmental delay, was able to play video games and to draw, and to say a few words; his comprehension and non-verbal communication were excellent, and he was the only child in our sample with toilet training; while he did not present frequent laughter, hyperphagia and skin picking were present; his OFC was above the $98^{\text {th }}$ percentile.

Patients 1- 4 were previously described in the report by Fridman et al. (2000a). Figure 1 shows patient $\#$.

Analysis of microsatellites within and outside the PWS/AS region performed in patients 5-8 disclosed isodisomy with the centromeric markers and reduction to homozygosity with the markers localized along the chromosome 15 in patient 5 , indicating a post-zygotic event. In patients $\# 7$ and $\# 8$, the centromeric markers showed heterodisomy; patient \#6 was non-informative. Three previously studied patients (\#s1, 2, and 4) presented isodisomy, with patients \#1 and \#4 showing reduction to homozygosity with all markers tested. Patient \#2 showed one crossover region, and patient \#3 was non-informative regarding the meiotic origin of non-disjunction (Fridman et al., 2000 a,b).

A summary of the clinical and behavioral characteristics of our UPD patients is presented in Table I.

\section{Discussion}

We reviewed the literature and compared the clinical data from 15 published UPD patients (Bottani et al., 1994; Freeman et al., 1993; Gillessen-Kaesbach et al.,1995; Malcolm et al., 1991; Nicholls et al., 1992; Prasad and Wagstaff, 1997; Smeets et al., 1992; Smith et al., 1997, 1998), including our own 4 patients, with data from our 21 deletion patients. In this comparison, we found other statistically significant clinical differences, besides those already described, i.e., delayed age-at-diagnosis, weight above the

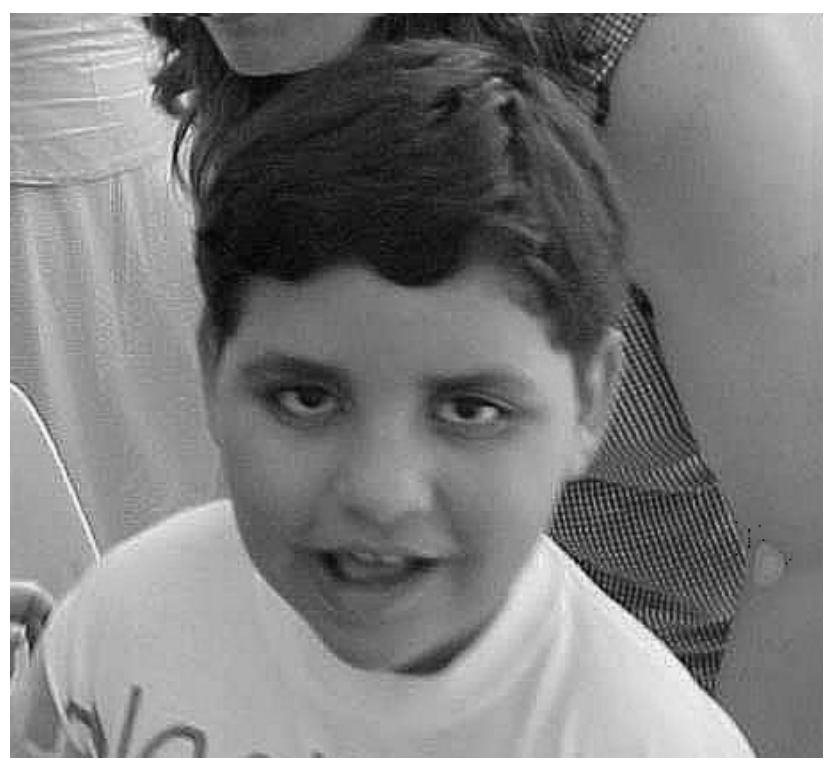

Figure 1 - Patient $\# 5$ at 7 years of age. 
Table I - Clinical features of AS patients with UPD.

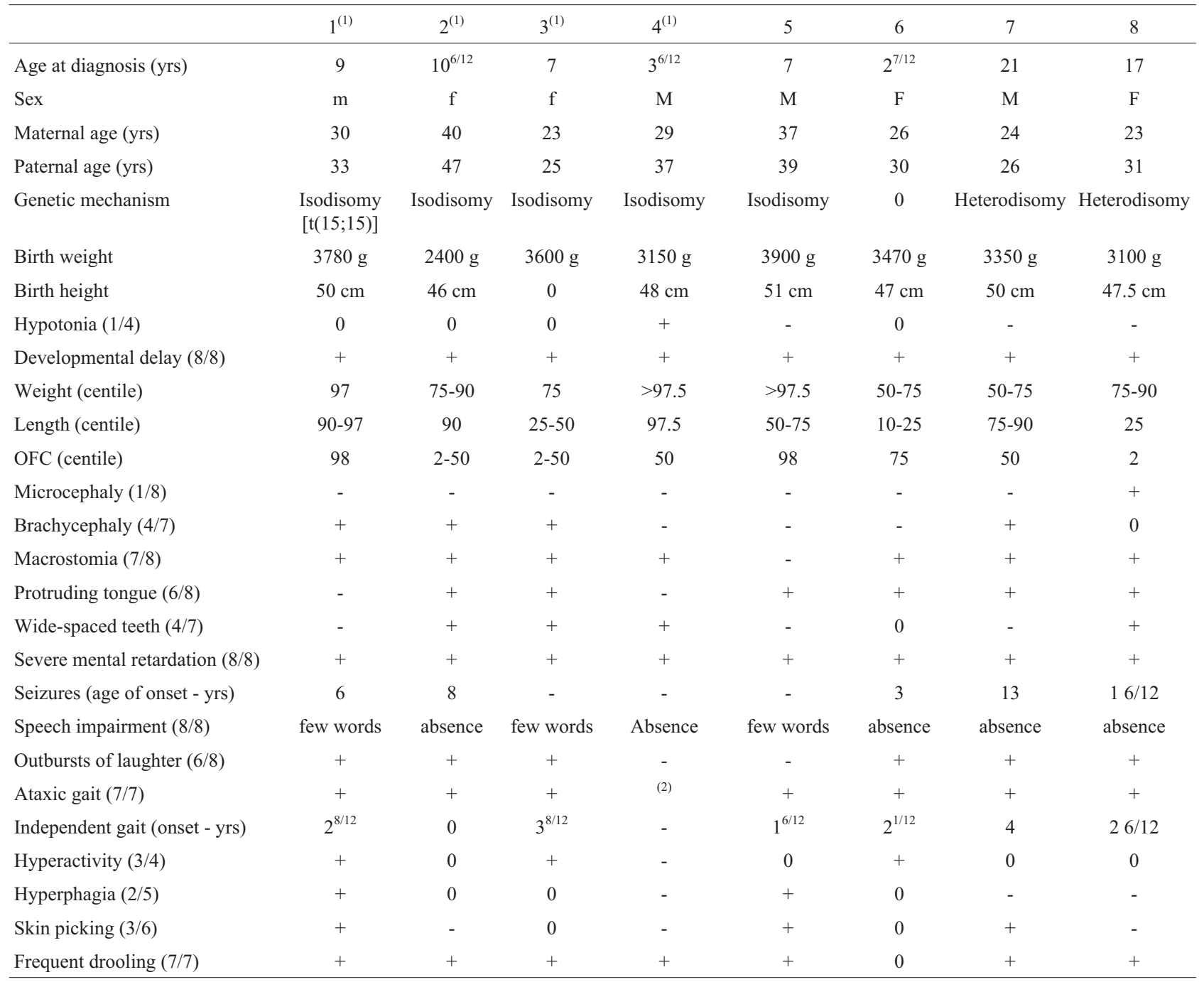

(2) - this patient did not walk at all.

$+=$ presence $-=$ absence $0=$ not known.

$75^{\text {th }}$ percentile, capacity to say a few words, and early walking in the UPD cases; prevalence of microcephaly, complete absence of speech, and earlier onset of seizures in the deletion group (Fridman et al., 2000a).

In this report, we describe the clinical and behavioral phenotypes observed within our UPD group (Table I). All 8 patients presented the common features of AS, such as developmental delay, mental retardation, ataxia, speech impairment and frequent drooling. However, only one had microcephaly, as opposed to patients \#1 and \#5, who had an OFC above the $98^{\text {th }}$ percentile. Patients $\# 1, \# 3$, and $\# 5$ were able to say a few words and to communicate by gestures. Only patients $\# 1, \# 2$, and $\# 7$ had late-onset seizures (at 6, 8, and 13 years of age, respectively). Patients \#4 and \#5 did not manifest frequent laughter. All but two patients showed weight above the $75^{\text {th }}$ percentile, and patients $\# 1$, \#2, and \#4 presented height above the $90^{\text {th }}$ percentile. Patients $\# 1$ and \#5 presented hyperphagia, and patients \#1, \#5, and \#7 showed skin picking, features which are common in the Prader-Willi syndrome. Patients \#1 and \#5 had no widespaced teeth. Patient $\# 5$ was very different from the others, as he had a better understanding and abilities to communicate, to play video games and to draw.

Previous reports have indicated that in patients with UPD the AS phenotype is milder, as compared to patients with deletions (Bottani et al., 1994; Freeman et al., 1993; Gillessen-Kaesbach et al., 1995; Smith e al., 1997), pointing out that children with UPD have a better physical growth, fewer or no seizures, less ataxia and higher cognitive skills. We suggest here that phenotypic and behavioral variability can also be found within the UPD group, and not only between the deletion and the UPD groups, since we found patients with features ranging from the typical AS phenotype and behavior to some of those also seen in PWS patients, and children with better communication and comprehension skills than usually seen in AS. 
In conclusion, we suggest that, in addition to the features previously appointed by us to be included in the clinical variability of AS (Fridman et al., 2000a), atypical patients as those with mental retardation, language impairment, happy disposition, and hyperactivity should be tested for AS by methylation analysis of SNRPN exon 1. Thus, the spectrum of phenotypic and behavioral characteristics in the Angelman syndrome seems to be broader than previously described.

\section{Acknowledgements}

This work was supported by FAPESP.

\section{References}

Angelman H (1965) "Puppet" children: a report on three cases. Develop Med Child Neurol 7:681-688.

Bottani A, Robinson WP, DeLozier-Blanchet CD, Engel E, Morris MA, Schmitt B et al. (1994) Angelman Syndrome due to paternal uniparental disomy of chromosome 15: A milder phenotype? Am J Med Genet 51:35-40.

Buiting K, Saitoh S, Gross S, Dittrich B, Schwartz S, Nicholls RD et al. (1995) Inherited microdeletions in the Angelman and Prader-Willi syndromes define an imprinting center on human chromosome 15. Nature Genet 9:395-400.

Clayton-Smith J and Pembrey ME (1992) Angelman syndrome. J Med Genet 29:412-415.

Dittrich B, Buiting K, Korn B, Richard S, Buxton J, Saitoh S et al. (1996) Imprinting switching on human chromosome 15 may involve alternative transcripts of the SNRPN gene. Nature Genet 14:163-170.

Driscoll DJ (1994) Genomic imprinting in humans. In: Friedmann T, (ed.) Molecular Genetic Medicine. Academic Press, New York, v. 4, pp. 37-77.

Freeman SB, May KM, Pettay D, Fernhoff PM and Hassold TJ (1993) Paternal uniparental disomy in a child with a balanced 15;15 translocation and Angelman syndrome. Am J Med Genet 45:625-630.

Fridman C, Varela MC, Kok F, Diament A and Koiffmann CP (2000a) Paternal UPD15: further genetic and clinical studies in four Angelman syndrome patients. Am J Med Genet 92:322-327.

Fridman C, Santos M, Ferrari I and Koiffmann CP (2000b) Further Angelman syndrome patient with UPD15 due to paternal meiosis II nondisjunction. Clin Genet 57:86-87.

Fridman C, Varela MC, Nicholls RD and Koiffmann CP (1998) Unusual clinical features in an Angelman syndrome patient with uniparental disomy due to a translocation $15 \mathrm{q} 15 \mathrm{q}$. Clin Genet 54:303-308.

Fryburg JS, Breg WR and Lindgren V (1991) Diagnosis of Angelman syndrome in infants. Am J Med Genet 38:58-64.

Gillessen-Kaesbach G, Albrecht B, Passarge E and Horsthemke B (1995) Further patient with Angelman syndrome due to paternal disomy of chromosome 15 and a milder phenotype. Am J Med Genet 56:328-329.

Kishino T, Lalande M and Wagstaff J (1997) UBE3A/E6AP mutations cause Angelman syndrome. Nature Genet 15:70-73.

Knoll JHM, Nicholls RD, Magenis RE, Graham Jr, JM, Lalande M and Latt AS (1989) Angelman and Prader-Willi syndromes shares a common chromosome 15 deletion but differ in parental origin of the deletion. Am J Med Genet 32:285-290.

Magenis RE, Toth-Fejel S, Allen LJ, Black M, Brown MG, Budden S et al. (1990) Comparison of the 15q deletions in Prader-Willi and Angelman syndromes: specific regions, extent of deletions, parental origin, and clinical consequences. Am J Med Genet 35:333-349.

Malcolm S, Clayton-Smith J, Nichols M, Robb S, Webb T, Armour JAL et al. (1991) Uniparental paternal disomy in Angelman's syndrome. The Lancet 337:694-697.

Malzac P, Webber H, Moncla A, Graham Jr, JM, Kukolich M, Williams C et al. (1998) Mutation Analysis of UBE3A in Angelman syndrome patients. Am J Hum Genet 62:1353-1360.

Matsuura T, Sutcliffe JS, Fang P, Galjaard R-J, Jiang Y-h, Benton CS et al. (1997) De novo truncating mutations in E6-AP ubiquitin-protein ligase gene (UBE3A) in Angelman syndrome. Nature Genet 15:74-77.

Mutirangura A, Greenberg F, Butler MG, Malcolm S, Nicholls RD, Chakravarti A et al. (1993) Multiplex PCR of three dinucleotide repeats in the Prader-Willi/Angelman critical region (15q11-13): molecular diagnosis and mechanism of uniparental disomy. Hum Molec Genet 2:143-151.

Nicholls RD, Pai GS, Gottlieb W and Cantú ES (1992) Paternal uniparental disomy of chromosome 15 in a child with Angelman syndrome. Ann Neurol 32:512-518.

Ohta T, Buiting K, Kokkonen H, McCandless S, Heeger S, Leisti $\mathrm{H}$ et al. (1999) Molecular mechanism of Angelman syndrome in two large families involves an imprinting mutation. Am J Hum Genet 64:385-396.

Prasad C and Wagstaff J (1997) Genotype and phenotype in Angelman syndrome caused by paternal UPD15. Am J Med Genet 70:328-329.

Robb SA, Pohl KRE, Baraitser M, Wilson J and Brett EM (1989) The 'happy puppet' syndrome of Angelman: review of the clinical features. Arch Dis Child 64:83-86.

Robinson WP, Kuchinka BD, Bernasconi F, Petersen MB, Schulze A, Brndum-Nielsen K et al. (1998) Maternal meiosis I non-disjunction of chromosome 15: dependence of the maternal age effect on level of recombination. Hum Mol Genet 7:1011-1019.

Saitoh S, Buiting K, Rogan PK, Buxton JL, Driscoll DJ, Arnemann J et al. (1996) Minimal definition of the imprinting center and fixation of a chromosome 15q11-q13 epigenotype by imprinting mutations. Proc Natl Acad Sci USA 93:7811-7815.

Smeets DFCM, Hamelm BCJ, Nelen MR, Smeets HJM, Bollen JHM, Smits APT et al. (1992) Prader-Willi syndrome and Angelman syndrome in cousins from a family with translocation between chromosomes 6 and 15. N Engl J Med 326:807-811.

Smith A, Marks R, Haan E, Dixon J and Trent RJ (1997) Clinical features in four patients with Angelman syndrome resulting from paternal uniparental disomy. J Med Genet 34:426-429.

Smith A, Robson L and Buchholz B (1998) Normal growth in Angelman syndrome due to paternal UPD. Clin Genet 53:223-225

Wajntal A, Moretti-Ferreira D, Souza DH and Koiffmann CP (1993) Cytogenetic evidence of involvement of chromosome regions $15 \mathrm{q} 12$ and $12 \mathrm{q} 15$ in conditions with associated overgrowth. DNA and Cell Biology 12:227-231. 